\title{
Towards an image of a memory trace
}

\author{
M. Inês Vicente and Zachary F. Mainen* \\ Champalimaud Neuroscience Programme, Instituto Gulbenkian de Ciência, Rua da Quinta Grande, Oeiras, Portugal \\ ${ }^{*}$ Correspondence: zach.igc@gmail.com
}

\section{A commentary on}

Associative and non-associative plasticity in Kenyon cells of the honeybee mushroom body

by Paul Szyszka, Alexander Galkin and Randolf Menzel

Learning and memory lead to functional and structural changes in the brain, ultimately providing a basis for adaptive behavior. The honeybee is an elegant model for the study of learning and memory formation as it permits both the visualization of neural activity related to the events occurring in olfactory learning and the behavioral assessment of olfactory learning (Galizia and Menzel, 2000). The formation of odor memories in the honeybee is thought to involve the two primary processing centers of the olfactory system, the antennal lobe (AL) and the mushroom body $(\mathrm{MB})$. The intrinsic neurons of the MB - the Kenyon cells (KCs), located within the lip region of the MB calyx - are the site of convergence of the neural pathways that transmit odor information from the projection neurons (PNs) of the AL and reward information from the VUMmx1 neuron (Hammer, 1997). In recent years, imaging studies performed in the honeybee $\mathrm{AL}$ and $\mathrm{MB}$ lip have indicated that pairing odor and reward induces changes in neural activity (Faber and Menzel, 2001; Faber et al., 1999), reinforcing the anatomical suggestion that KCs are likely to undergo associative plasticity during learning.

This study, Szyszka et al. (2008) provides new evidence that odor-evoked activity of KCs can be modified by sensory experience, and the changes can be associative or nonassociative. Szyszka et al. (2008) visualized odor representations in the $\mathrm{MB}$ of the honeybee by imaging the calcium responses of a subpopulation of KCs - the clawed KCs, by retrogradely labeling the cell population using dye injections into the ventral alpha/gamma lobe. This allowed the authors to image, in a relatively selective way, the bulk dendritic activity of KCs over neuropil. Szyszka et al. (2008) first examined KC activity in response to repeated stimulation with the same odor and found that it led to a decrease in $\mathrm{KC}$ responses, a non-associative form of plasticity resembling a phenomenon previ- ously described in locust PNs (Stopfer and Laurent, 1999). Separate experiments found that honeybee PNs did not show repetitioninduced depression, indicating that the effect is not a generalized run-down. The authors then compared KC activity before and after pairing one odor with sucrose reward and tested how the associative pairing procedure affects $\mathrm{KC}$ responses to the rewarded odor $(\mathrm{CS}+)$ and to the unrewarded odor (CS-). During pre-training, KC responses to both CS+ and CS- decreased with repeated stimulation. After pairing CS+ with sucrose CS+ responses recovered and CS- responses decreased further (Figure 1). Intriguingly, examination of spatiotemporal patterns within the imaged region before and after conditioning suggested additional changes in both CS+ and CS- evoked KC activity following conditioning.

The results of this study support the suggestion that KC odor responses can be modified by sensory experience and that these changes can be associative, extending the earlier work of the Menzel group (Faber and Menzel, 2001; Faber et al., 1999). Although a causal link between this activity and learn-

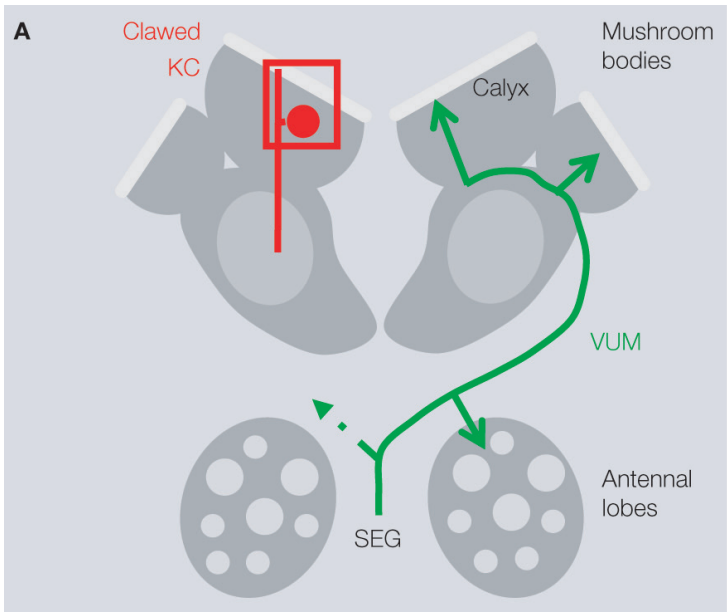

Figure 1 | $\mathrm{KC}$ responses are subject to associative and non-associative plasticity. (A) Scheme of honeybee antennal lobes and mushroom bodies (MB). Calcium responses in the somata and dendrites of clawed Kenyon cells (clawed KCs, red) were recorded in the MB calyx (the square indicates the imaged area). The VUMmx1 neuron (green), which connects the

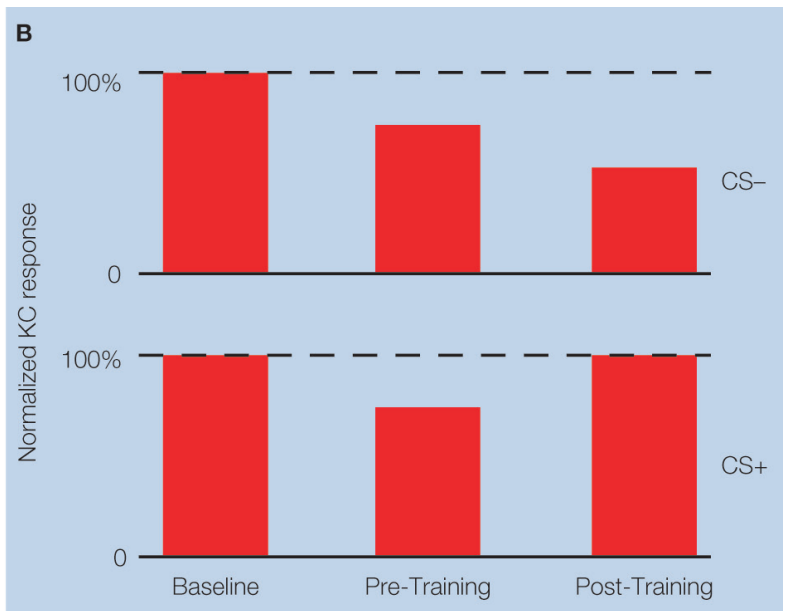

subesophageal ganglion (SEG) with the antennal lobes and the MB calyces, mediates the reinforcing function of the reward. (B) Amplitude of $\mathrm{KC}$ responses to the rewarded odor ( $\mathrm{CS}+$ ) and to the unrewarded odor (CS-) before training, after repeated odor stimulation with both odors (pre-training), and after pairing $\mathrm{CS}+$ with sucrose (post-training). 
ing and memory remains to be established, the strengthening of support for a leading candidate cellular substrate is an encouraging observation that can be followed up in several ways. Firstly, it should be possible to examine the same cell population at the molecular and cellular levels in order to elucidate the processes underlying these modifications. Secondly, the changes in spatiotemporal patterns hint at restructuring of $\mathrm{KC}$ odor representations that might reflect a correlate of information storage. It may now be feasible, using similar imaging approaches but at single cell resolution, to resolve the details of the changes. Finally, it should be possible, albeit technically challenging, to take advantage of the possibility of behavioral measurements in combination with imaging to try to directly link KC changes to memory storage itself.

\section{REFERENCES}

Faber, T., Joerges, J., and Menzel, R. (1999). Associative learning modifies neural representations of odors in the insect brain. Nat. Neurosci. 2, 74-78.

Faber, T., and Menzel, R. (2001). Visualizing mushroom body response to a conditioned odor in honeybees. Naturwissenschaften 88, 472-476.

Galizia, C. G., and Menzel, R. (2000). Odour perception in honeybees: coding information in glomerular patterns. Curr. Opin. Neurobiol. 10, 504-510.
Hammer, M. (1997). The neural basis of associative reward learning in honeybees. Trends Neurosci. 20, 245-252.

Stopfer, M., and Laurent, G. (1999). Short-term memory in olfactory network dynamics. Nature 402, 664-668.

Szyszka,P., Galkin,A., and Menzel, R. (2008).Associative and non-associative plasticity in Kenyon cells of the honeybee mushroom body. Front. Syst. Neurosci. 2, 3 .

Received: 20 October 2008; published: 15 December 2008

Citation: Front. Neurosci. (2008) 2, 2: 131-132. doi: 10.3389/neuro.01.041.2008

Copyright: (c) 2008 Vicente and Mainen. This is an openaccess publication subject to an exclusive license agreement between the authors and the Frontiers Research Foundation, which permits unrestricted use, distribution, and reproduction in any medium, provided the original authors and source are credited.

\section{Brain rhythms in the human medial temporal lobe}

\section{Hans-Jochen Heinze*}

Department of Neurology, Otto-von-Guericke-University, Magdeburg, Germany

* Correspondence: hans-jochen.heinze@med.ovgu.de

\section{A commentary on}

Independent delta/theta rhythms in the human hippocampus and entorhinal cortex

by Florian Mormann, Hannes Osterhage, Ralph G. Andrzejak, Bernd Weber, Guillén Fernández, Juergen Fell, Christian E. Elger and Klaus Lehnertz

Ever since the discovery of the electroencephalogram (EEG), brain oscillations have been a major focus of neuroscientific research. The perpetual interactions among multiple network oscillators enable the brain to perform global computations on multiple spatial and temporal scales. A prominent example of interacting oscillations is the phenomenon of phase precession of place cells in the rodent hippocampus (O'Keefe and Recce, 1993). When the rat traverses a particular region in its environment, these cells fire periodic bursts at a slightly faster rhythm than the ongoing theta oscillation in the hippocampus. The firing thus occurs at increasingly early phase angles of the local field oscillations, and the spatial position of the animal can be decoded from these phase angles.

In this issue, Mormann et al. (2008) investigate oscillatory activity in the human hippocampus and in its main input structure, the entorhinal cortex. Using a unique data set of intracranial EEG signals directly recorded from the healthy medial temporal lobes of epilepsy patients with strictly unilateral seizure onset, the authors perform an elegant data analysis and provide evidence for the existence of independent theta generators in the human hippocampus and entorhinal cortex.

Of these two independent theta rhythms, the entorhinal rhythm is seen as being mediated by sensory and other cortical inputs, whereas the hippocampal rhythm is assumed to reflect theta activity autonomously generated within the hippocampus itself, mediated by inputs from the medial septum. Furthermore, the authors consider the hypothesis that the two independent rhythms represent the two oscillators of slightly different frequencies postulated by the interference model of theta phase precession (O'Keefe and Recce, 1993). Recent advances in recording technology that allow simultaneous recording of local field potentials and single-neuron activity in humans make this hypothesis an exciting and, more importantly, a testable one.

Previous studies on intracranial EEG recordings have shown that successful memorization of presented words is associated with an increase in entorhinalhippocampal theta coherence (Fell et al.,
2003). Mormann et al. now conjecture that the independent theta rhythms found in the entorhinal cortex and hippocampus may need to be actively synchronized to facilitate the synaptic plasticity involved in memory encoding. This hypothesis is backed by findings that the theta phase at which an action potential arrives in the rodent hippocampus determines the direction of plasticity, i.e. whether it results in long-term potentiation or depression. Synchronization of theta activity between the hippocampus and entorhinal cortex could thus create a slowly modulated facilitating state that provides a temporal basis for encoding or retrieval of separate items. A potential mechanism to generate the synchronization was discovered by Mormann et al. (2005). In a continuous word recognition paradigm, the authors found that the presentation of a visual stimulus causes a simultaneous phase reset of ongoing oscillatory activity in the hippocampus and entorhinal cortex.

Furthermore, the existence of independent theta rhythms plays an important role for a number of mechanisms related to gamma oscillations (Fries et al., 2007), as gamma activity has been found to be coupled to the phase of ongoing theta oscillations (Canolty et al., 2006; Demiralp et al., 2007; Mormann et al., 2005). 